\title{
La función docente como humanización del tiempo inhumano en la formación jurídica
}

\section{The teacher function as humanization of the inhuman time in legal formation}

Jose Miguel Camacho Castro (c) Magíster en Derecho con énfasis en teoría del derecho. Docente investigador de la Universidad La Gran Colombia Seccional Armenia.

Cómo citar: Camacho, J.M.(2016) La función docente como humanización del tiempo inhumano en la formación jurídica.Sophia 12 (2): 271-278.

\section{Resumen}

El avance de la tecnología y la globalización han propiciado la aceleración de la vida humana en general. La producción de conocimiento se ha vinculado a la misma dinámica, lo cual ha conducido a que los acontecimientos en materia educativa -en especial la producción de material bibliográfico- vayan más rápido que lo que puede ir cualquier cerebro humano: de allí que se hable, según Peter Sloterdijk, de tiempo inhumano. Esta situación puede generar desasosiego en cualquier ser humano en formación, pues el proceso puede exigirle más de lo que fisiológicamente puede dar. Este artículo se centra en el papel que el docente desempeña en evitar el desasosiego que la situación descrita produce o puede producir. El docente opera a modo de filtro del tiempo, que permite al ser humano en formación la preparación suficiente para afrontar un mundo que irá siempre delante de él.

Palabras clave: Educación superior, formación jurídica, Peter Sloterdijk, tiempo, tiempo inhumano.

\begin{abstract}
Technology and globalization progress have promoted acceleration of human life in general. Knowledge production has involved to the same dynamics, which has led education events to go faster than any human brain can do: this fact results in speaking about inhuman time. This situation may cause uneasiness in any under-formation human being, since the process may require him more than what he physiologically can give. This article centers in the role performed by the teacher to prevent uneasiness which the above described situation causes of may cause. The teacher operates as a filter of the time, which allows the human being in formation sufficient preparation to face a world that will always go ahead of him.
\end{abstract}

Keywords: Higher education, legal formation, Peter Sloterdijk, time, inhuman time. 


\section{Introducción}

La técnica ha propiciado la aparición de tiempos inhumanos. Con esto se quiere decir que el ritmo en que ocurren los eventos es en extremo veloz; tanto así que un ser humano normal no puede aprehenderlo en su totalidad, de tal modo que cuando comprende un hecho a cabalidad, o cuando se lanza a su análisis o interpretación, ya otros mil han entrado en escena modificando su panorama sensorial. La globalización ha sido un vehículo para que el tiempo devenga en una variable inhumana: la información, los productos y el dinero fluyen a, literalmente, la velocidad de la luz a través de cables de fibra óptica diseminados por el mundo entero.

La academia no ha sido ajena ni a la globalización ni a la inhumanización del tiempo: los avances científicos, tecnológicos y conceptuales se presentan a un ritmo $\tan$ acelerado que apenas puede uno enterarse. Stiglitz (2002) afirma que la globalización académica es uno de los aspectos más saludables de la globalización. Genera perplejidad que esto sea apenas parcialmente cierto, pues tal vez a investigadores de alto nivel este fenómeno les venga muy bien; pero a los que se encuentran en formación y a estudiantes, esta situación de flujo infinitamente amplio e infinitamente veloz de información y avances puede producirles más bien desorientación, desasosiego.

Este problema solo puede ser atajado por la figura del docente. En sus funciones de selección -tanto de contenidos como de metodologías- y especialización, se vislumbra otra función de mayor peso: la de humanizar el tiempo que ha devenido inhumano por la técnica y la globalización, para crear un espacio de sosiego en el cual los seres humanos que apenas inician su formación puedan aprehender herramientas que, más adelante, le permitirán enfrentarse e insertarse con éxito en el inevitable tiempo inhumanizado.

En el campo jurídico la situación es especialmente compleja. Solo para el caso colombiano el volumen de información es prácticamente intratable. Entre 1992 y 2015 se expidieron en Colombia 1771 leyes, es decir, 77 leyes por año en promedio, una ley cada 4 días. En el mismo período la Corte Constitucional expidió 23442 sentencias, esto es, 1019 sentencias por año en promedio, casi tres sentencias diarias. $\mathrm{La}$
Corte Constitucional es solo uno de los 4 máximos tribunales del país. Aún no se habla de la rama ejecutiva del poder público, que solo en 2015 expidió aproximadamente 2500 decretos presidenciales, o sea, más de 6 decretos por día, uno cada 4 horas. Aún no se contabilizan aquí las circulares, conceptos, resoluciones, directivas, autos, y demás instrumentos jurídicos que contienen derecho vigente y son, por ende, parte del objeto de estudio del derecho. No aparece aún aquí reseñada la producción bibliográfica doctrinal, comentarios a jurisprudencia, artículos científicos que son igual e incluso más relevantes que todo lo mencionado hasta ahora.

Lo anterior muestra que en el campo jurídico es especialmente relevante la función docente como filtro de humanización de tiempos inhumanos, es decir, de tiempos que no pueden ser captados, aprehendidos en su totalidad por un cerebro humano normal. La especialización, experiencia y capacidad de selección -temática y metodológica- del docente son herramientas de ese filtro que favorecen la creación de un espacio de sosiego que permite a los estudiantes adquirir herramientas básicas para posteriormente insertarse en la comprensión del tiempo inhumano que prevalece en la actualidad.

\section{Tiempo inhumano en el derecho}

\section{La tecnología y los seres humanos}

La forma en que se valora el papel de la tecnología en una sociedad es un tema muy importante. Por ejemplo, la hipótesis de la "tecnología malvada" considera que prácticamente ningún avance en este campo es positivo y que siempre los efectos de las nuevas tecnologías son impredecibles y peligrosos y 'malvados' (Mattei \& Russi, 2012; Heath \& Potter, 2005: 327-361). Incluso hay formas más radicales de ver el asunto, hasta prácticamente satanizar cualquier avance y solo señalar la permanente "[...] vulnerabilidad de la civilización hipertecnológica" (Riechmann, 2006: 450).

El desarrollo técnico del siglo XX y lo que va del XXI no solo ha posibilitado la destrucción masiva de millones de vidas humanas, sino que ha traído inmensa cantidad de efectos positivos; en efecto, la balanza de la historia se inclina a favor de la ciencia, 
la técnica y la tecnología. Si bien es cierto que los desarrollos técnicos dieron la posibilidad de fabricar variopintas bombas atómicas, también propiciaron la aparición de salas de cirugía, antibióticos y otros beneficios que han extendido la duración y calidad de la vida humana (Russell, 1961).

Sostener estas visiones tan negativas alrededor del avance tecnológico es complicado en el marco de las posibilidades que las propias tecnologías brindan. El acceso a internet ha sido uno de los hitos más importantes de la historia de la humanidad y todavía se esperan avances más significativos. Sloterdijk (2014), afirma que la humanidad ya acaricia las fronteras de avance de las tecnologías de la información, pues solo hace falta la directa trasmisión de pensamientos. Castells (2001) considera que las redes informáticas han ayudado desde múltiples frentes y en distintos puntos geográficos a la consolidación de la democracia y la libertad. Basta, en todo caso, con pensar en las posibilidades cognitivas que pueden ofrecer tres mil o cuatro mil millones de cerebros que en breve -se estima que para el 2020- podrán acceder a los contenidos de la red (Diamandis, 2012). Las posibilidades son vastísimas.

\section{Las velocidades humanas y la velocidad tecnológica}

La tecnología de la información ha propiciado una gran aceleración de la producción en todos los niveles. Desde el sector financiero, en el cual millones de transacciones de gran complejidad y riesgo se realizan en apenas unos cuantos segundos -a veces defraudando a terceros- (Lewis, 2014; Camacho, 2013) hasta el mundo del conocimiento, en el cual diariamente se producen miles de documentos académicos y científicos y se amplía la frontera del conocimiento minuto a minuto. La tecnología de la información, a través del vehículo de la globalización potenciado por ella misma, ha logrado que los procesos de creación y comunicación humana se hagan en una escala inhumana, i.e., que no es posible hacerse un cuadro completo del conocimiento que los seres humanos producimos a diario, lo cual puede generar perplejidad en los procesos de educación de todos los niveles.
La globalización propiciada por la información que fluye por el mundo gracias a la tecnología supone la necesidad de "[...]construir y reconstruir la sociedad del saber y de la cultura" (Beck, 2008: 255). La globalización implica que el conocimiento pasa a ser central dentro de la dinámica humana, resignificando la idea de trabajo. De allí que los procesos de generación, transmisión y socialización del conocimiento se hayan acelerado como en el caso de la industria y los servicios financieros. Está aceleración del conocimiento, aunque puede verse como el efecto más positivo de la globalización (Stiglitz, 2002) puede ser fuente de perplejidades en el ámbito de la formación universitaria.

Esta aceleración ha sido tal que puede ser tan icónica como la que se produjo con el origen de la cultura escrita misma. Sloterdijk $(2010 ; 2012)$ describe el proceso a través del cual la humanidad se escindió en dos: por un lado, están aquellos que permanecen en el río del hábito y por otro, aquellos que salen del río hacia el descubrimiento y la consecuente perturbación del hábito. Surge de allí una vieja comunidad, habituada y pasiva y, además, surge una élite dedicada a aprender y a ejercitarse. Este autor lo denomina «tiempo axial», afirmando:

El efecto del «tiempo axial» consistiría, por ello, no tanto en un interés que aparece de repente a nivel planetario por una espiritualización elevada, sino que habría surgido de la enorme perturbación del hábito que siguió al descubrimiento, posible desde el puesto de la orilla, de las inercias encarnadas en el ser humano. Habría sido responsable de esto -en su parte más importante- la aceleración interna desencadenada por las primeras culturas escritas, que a su vez sería responsable de que los cerebros de quienes escribían dejaran atrás el hábito de los que no escribían (de forma análoga a como el cuerpo de los ascetas, atletas y acróbatas superan las capacidades de los cuerpos de las personas ordinarias). (Sloterdijk, 2012: 251).

La tecnología informática y principalmente la globalización del conocimiento han creado tal aceleración que ha facilitado una nueva escisión: aquellos que, con altos niveles de formación, pertenencia a redes académicas especializadas, acceso preferente a publicaciones -en papel o de forma virtual- de importantes centros de producción de conocimiento, van siempre un paso adelante; 
y otros que simplemente no tienen ese tipo de acceso. Buena parte de la comunidad académica a nivel internacional pertenece al segundo grupo. Lo preocupante es que incluso en el centro -siguiendo las ideas de centro-periferia- (Wallerstein, 2006) puede surgir perplejidad en aquellas personas en formación.

De hecho, "[...]hemos entrado en una era de velocidades inhumanas; $y$ ello atraviesa todas nuestras historias vitales. Participamos de una aceleración desmedida y solo poseemos un presentimiento confuso de lo que realmente sucede con nosotros" (Sloterdijk, 2014:140, 141). En el mismo sentido, Pedro Salinas afirma que

[...] es un hecho que la copiosidad creciente de material impreso [ni hablar del virtual propiciado por las modernas tecnologías de la información] que solicita a diario nuestra atención y nos hace llamadas a gritos -los colorines de las portadas chillonas- desde los escaparates, coloca al hombre culto moderno en un apuro: ¿cómo entendérselas con esa multiplicidad? (Salinas, 1995:109).

La aceleración del conocimiento en general no es ajena, naturalmente, al conocimiento jurídico. Podría decirse incluso que la situación para la formación jurídica es especialmente delicada por la inmensa variabilidad de la información, el constante ir y venir en distintos sentidos por parte de las autoridades públicas que, en la formulación de normas y políticas públicas, alteran permanentemente el objeto de estudio de un jurista en formación.

\section{La inhumana velocidad jurídica en Colombia}

Con el concepto de velocidad inhumana se quiere señalar aquí la existencia de un ritmo de avance tan veloz en el conocimiento y en la información que no puede ser aprehendido por un cerebro humano normal. En ocasiones ni siquiera es viable valorar la velocidad de tal avance. Un decente grado de actualización, un nivel mínimo de formación, supone en la actualidad esfuerzos sobrehumanos que se pueden tornar -a menudo lo hacen- imposibles, patológicos, degradantes o cuando menos, intranquilizantes.

Un estudiante de derecho en Colombia en cualquier nivel de formación se enfrenta a un panorama sumamente amplio en lo que a generación de conocimiento se refiere. Como se indicaba en la introducción, las cifras son perturbadoras. Como se muestra en la gráfica 1 (ver sig pág), solo la Corte Constitucional ha expedido, desde el año de su creación en 1991 y hasta el 2015, 23426 sentencias, entre sentencias de constitucionalidad, de tutela y de unificación.

Hay que anotar que las sentencias no son los únicos documentos relevantes que expide la Corte Constitucional, sino que faltan, por ejemplo, los autos de seguimiento al cumplimiento de sentencias, documentos que garantizan que ciertos fallos de trascendencia nacional -como el fallo que ordenó proteger a las personas en situación de desplazamientose reflejen efectivamente en la realidad.

Los autos expedidos por la Corte Constitucional, como se muestra en la gráfica 2 (ver sig pág), son documentos que están en franco ascenso numérico, pues su importancia es cada vez más significativa. Por ejemplo, la intervención de la Corte en el asunto del desplazamiento forzado, realizada a través de una sentencia y varias decenas de autos de seguimiento, ha generado por lo menos seis efectos positivos para la solución del problema: el efecto creador, el efecto de desbloqueo institucional, el efecto coordinador, el efecto deliberativo, el efecto de política pública y el efecto social (Rodríguez \& Rodríguez, 2010).

Con las anteriores cifras se pretende mostrar que con la sola producción de la Corte Constitucional es suficiente para el desánimo: comprender a cabalidad el papel de esta institución no toma los pocos años de formación, sino que puede tomar la vida entera.

Naturalmente, para un estudiante de derecho en cualquier nivel (pregrado o posgrado) no basta con tener una decente comprensión del papel de la Corte Constitucional en Colombia. Se requiere tener conocimiento de la Constitución Política de Colombia, documento amplio e impreciso, sujeto a variopintas interpretaciones por su formulación altamente valorativa; de la producción jurídica de los otros tres tribunales supremos (Corte Suprema de Justicia, Consejo de Estado y Consejo Superior de la Judicatura); de las leyes expedidas por el Congreso de la República antes y después de 1991 (entre 1992-2015 fueron 1771) con sus respectivas vigencias y derogaciones; de los decretos reglamentarios expedidos por el Presidente de la República y sus 
Grafica 1. Producción Jurisprudencial de la Corte ConstitucionalNúmero de Sentencias / Año

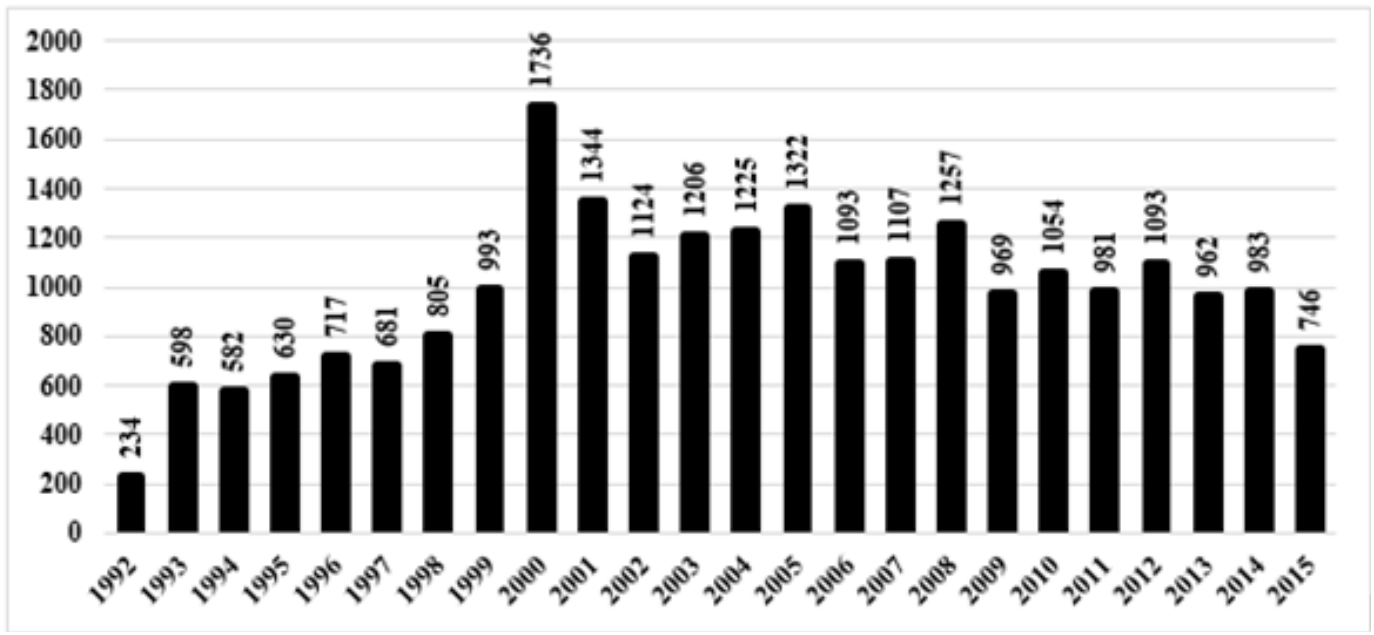

Fuente: Corte Constitucional de Colombia (2016)

Grafica 2. Autos expedios por la Corte Constitucional

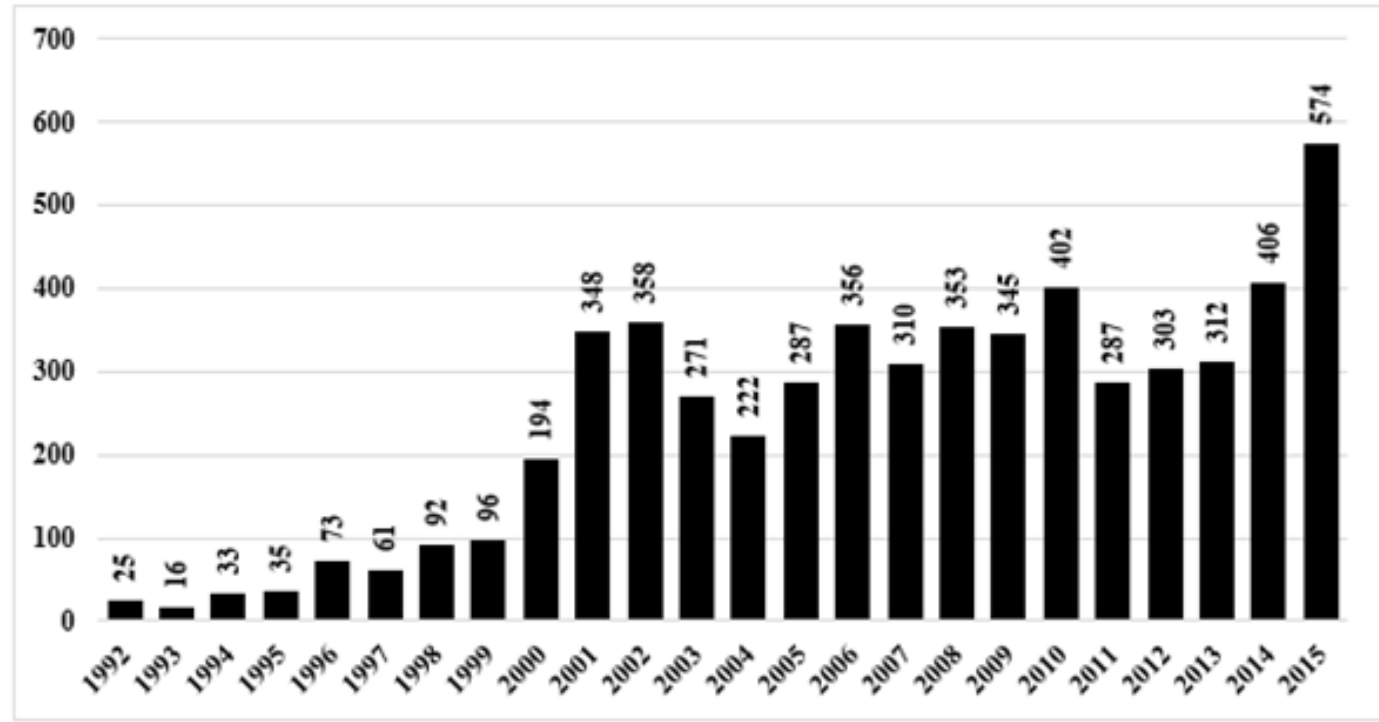

Fuente: Corte Constitucional de Colombia (2016) 
ministros (que regulan la forma de aplicación de las leyes); de las resoluciones, circulares y sentencias expedidas por las superintendencias (órganos de control sobre actividades de todo tipo, como los servicios públicos o la competencia empresarial); de la doctrina nacional y extranjera (comentarios eruditos sobre la ley y la jurisprudencia); y de la producción científica relacionada (artículos científicos y libros resultado de investigación, entre otros).

Se podría describir la situación con más detalle, mostrando al lector infinidad de cifras que se encaminan a ilustrar que la formación jurídica es puro desasosiego. No es posible valorar con exactitud el alcance de esta divergencia entre lo que un ser humano en formación es capaz de aprender y lo que hay por aprender. Es más difícil aún tener una idea de la tasa de crecimiento de esta divergencia, de esta brecha, de este acantilado inmenso al que no todos le asignan el mismo valor que Sócrates alguna vez le dio a la sensación de vacío e ignorancia como venero del fervor por el conocimiento y el autoconocimiento (Cornford, 2011).

\section{La función docente como filtro del tiempo inhumano}

\section{El estudiante ciego}

En El poema de los dones, Jorge Luis Borges describe la paradoja de tener a su disposición cerca de 800.000 volúmenes y ser ciego (Borges, 1998). Con toda aquella información, con aquellos inmensos anaqueles repletos de oferentes lomos al alcance de la mano, Borges, ya ciego, no podía hacer nada. Quizá un estudiante cualquiera esté en la misma situación. Quizá Borges, cuando aún veía más que sombras, reconocía que el tiempo le quedaría corto para leer cuanto había por leer -o para releer cuanto quería releer- (Borges, s.f.).

Tal vez un estudiante de derecho puede tener todas las herramientas a su alcance, preciarse de una inteligencia privilegiada, hacer gala de la más afinada actitud crítica y ser el pensador más autónomo (Wong, Peña \& Falla, 2016), y aun así estar ciego, como ante un muro, en frente de las estanterías de una biblioteca o de una pantalla de computador. Esta ceguera viene producida por el vasto océano de información y conocimiento que hace perder el enfoque ${ }^{1}$. Ciertos rasgos de la actividad docente en el ámbito universitario, como la selección y la especialización, permiten el filtro del tiempo inhumano, en una suerte de gestión de la libertad.

\section{Una plaza llena de fuentes}

Imagínese en este punto una plaza amplísima con un número desconocido de fuentes de las que brotan líquidos de distintos tipos. Solo es posible obtener conocimiento de esas sustancias bebiéndolas. Si se bebe sin orden, de aquí y de allá, se termina intoxicado. Supóngase, a la vez, que esa plaza tiene un guía, el cual no conoce las propiedades de todas las sustancias líquidas que brotan de las fuentes y ni siquiera sabe cuántas fuentes hay. Pero su experiencia y su especialización le permiten tener ciertas precauciones para evitar intoxicaciones.

Cuando el guía -docente universitario- selecciona de entre las fuentes que conoce varias bebidas para darlas a sus visitantes, ¿qué es exactamente lo que hace? Bajo lo expuesto hasta ahora solo hace una cosa: evitar la intoxicación. Imprimir calma al proceso, cierto orden. No siempre podrá evitarse la intoxicación, pues no todos los visitantes -estudiantesson iguales. Pero aquí el docente opera a la manera de un filtro que, en lugar de mostrar la plaza entera a sus visitantes, la suministra de a poco y con cuidado, protegiéndolos de una intoxicación y midiendo la capacidad de absorción de cada uno de ellos.

De manera que el docente acerca a sus estudiantes lo que él cree que no les intoxicará, pero bajo esta interpretación, este 'acercar' no es una fase final, sino apenas intermedia. El guía nunca oculta la existencia de miles o millones de fuentes, simplemente advierte que hay que ir con cuidado para impedir una intoxicación. Luego de esos primeros acercamientos, pueden los visitantes empezar a probar por sí mismos las sustancias que brotan de las fuentes, pues ya han desarrollado cierta resiliencia, cierta sana inquietud.

Además, si el proceso de leer es "[...]encontrar, entre lo que está cerca, aquello que puede usarse para sopesar y reflexionar" (Bloom, 2000: 24), entonces es necesario que medie alguien o algo que

1. Hay que recordar aquí las palabras de Gabriel Zaid (2014, p. 15): "Los libros se publican a tal velocidad que nos vuelven cada día más incultos. Si alguien lee un libro diario, deja de leer 6.000 publicados el mismo día. Sus libros no leídos aumentan 6.000 veces más que sus libros leídos. Su incultura, 6.000 veces más que su cultura". 
ponga cerca aquello que después será usado para sopesar o reflexionar. Esa acción de poner cerca, posible únicamente gracias a la especialización y a la selección realizada por los docentes, es la única forma a través de la cual puede el estudiante en general y el estudiante de asuntos jurídicos en particular, salvarse de ser aplastado o de, literalmente, perder la cabeza por el enorme volumen de producción de conocimiento en el mundo globalizado y altamente tecnificado.

\section{Poner cerca: humanizar el tiempo inhumano}

En la mitología griega, Prometeo da el fuego a los hombres. Si en lugar de eso nos hubiese dado una herramienta terminada, todo giraría en torno al martillo o el arco, y el devenir humano hubiese sido distinto. El fuego permitió aprender a vivir por sí mismo al hombre, le dio libertad, le permitió explorar y luego, fabricar más herramientas. ¿También lo haría el martillo?

La elección supuesta que aquí se propone para Prometeo es la elección que a diario hacen los docentes. Ninguna selección resulta neutra, por más que las pretensiones de formación sean transparentes. El acto de poner cerca evita que el estudiante se indigeste en el proceso de abordaje del mundo del conocimiento. El mismo acto de poner cerca es eminentemente metodológico, pues no pretende la trasmisión de contenidos, sino enseñar a no intoxicarse, a ir paso a paso, a tener paciencia, a esperar lo suficiente.

Este poner cerca es, se reitera, un filtro que la especialización y el ejercicio de selección del docente hace del tiempo que, por la técnica y la globalización, ha devenido inabarcable para seres humanos normales. Un filtro que permite al estudiante tener un espacio de sosiego que le permita iniciar y afinar su proceso de formación. Usando la metáfora de Carroll (2009), leer es como comer. La función docente como filtro del tiempo inhumano es también enseñar a nutrir el cerebro adecuadamente, antes que nutrirlo directamente.

\section{Conclusiones}

Las posibilidades técnicas gestadas durante el siglo XX y potenciadas hasta ahora durante el siglo XXI han aumentado la velocidad de producción en todos los frentes. El área del conocimiento no es la excepción. El volumen de información que se produce, de libros, artículos y otros documentos es tan alto, que para una persona en formación puede representar una peligrosa fuente de preocupación, que puede llevar a la frustración de su proceso educativo. En el campo de la formación jurídica esta situación es especialmente delicada si se tiene en cuenta que el devenir político e institucional altera de forma constante el objeto de estudio de la ciencia jurídica.

Lo que Sloterdijk describe como tiempos inhumanos hace referencia a procesos de aceleración desmesurada en la producción de conocimiento e información que ocasiona que ningún ser humano con un cerebro normal pueda estar al tanto, medianamente informado, medianamente al día. Por esto, todo intento de formación supone esfuerzos cada vez menos realizables, por no decir imposibles. Esto hace que el papel de estudiante, tanto en pregrado como en posgrado -investigadores jóvenes- sea complicado en grado sumo y en muchas ocasiones desilusionante, frustrante.

Esta situación de posible desasosiego -o de franco desasosiego- tiene como canalizador el rol docente. Una reinterpretación de la función docente a la luz del reconocimiento del hecho de la velocidad inalcanzable, conduce a ver el docente como un sujeto que pone cerca la información; que selecciona a través de su especialización y su experiencia aquel mundo hiperveloz; que impide que el ser humano en formación se indigeste con el volumen inmenso del conocimiento; que construye paso a paso la libertad del estudiante para utilizar en sus reflexiones lo que se le ha puesto cerca de antemano.

\section{Referencias bibliográficas}

Beck, U. (2008). ¿Qué es la globalización? Falacias del globalismo, respuestas a la globalización. Barcelona, España: Ediciones Paidós Ibérica S.A. 
Bloom, H. (2000). Cómo leer y por qué. Bogotá D.C., Colombia: Editorial Norma S.A.

Borges, J.L. (s.f.). El libro. Tomado de: http://www. laserpblanca.com/borges-el-libro

Borges, J.L. (1998). Obra poética, 2 (1960-1972). Madrid, España: Alianza Editorial.

Camacho, J.M. (2013). Un mundo riesgoso: sobre contratos derivados y crisis económicas. Inciso, 15 (1), 103-116.

Castells, M. (2001). La Galaxia Internet. Barcelona, España: Plaza y Janés Editores S.A.

Carroll, L. (2009). Alimentar la mente. Madrid, España: Gadir.

Cornford, F.M. (2011). Antes y después de Sócrates. Barcelona, España: Editorial Planeta S.A.

Corte Constitucional de Colombia. (2016). Estadísticas 1992-2016. Tomado de: http:// www.corteconstitucional.gov.co/relatoria/

Diamandis, P. (2012). Abundance is our future. Tomado de: http://www.ted.com/talks/ peter_diamandis_abundance_is_our_future

Heath, J. \& Potter, A. (2005). Reberlarse vende. Bogotá D.C., Colombia: Aguilar, Altea, Taurus, Alfaguara S.A.

Lewis, M. (2014). Flash Boys. La revolución de Wall Street contra quienes manipulan el mercado. Bogotá D.C., Colombia: Editorial Planeta S.A.

Mattei, U. \& Russi, L. (2012). The evil technology hypothesis: a deep ecological reading of international law. Cardozo Law Review de novo, (263), 263-277.

Riechmann, J. (2006). Ciencia, tecnología y responsabilidad socioecológica: algunos hitos históricos (1945-2005). En J. Riechmann (ed.). Perdurar en un planeta habitable. Ciencia tecnología y sostenibilidad (pp.441-463). Barcelona, España: Icaria Editorial S.A.
Rodríguez, C. \& Rodríguez, D. (2010). Cortes y cambio social. Cómo la Corte Constitucional transformó el desplazamiento forzado en Colombia. Bogotá D.C., Colombia: Centro de Estudios de Derecho, Justicia y Sociedad, Dejusticia.

Russell, B. (1961). El impacto de la ciencia en la sociedad. Madrid, España: Aguilar S.A.

Salinas, P. (1995). El defensor. Bogotá D.C., Colombia: Editorial Norma S.A.

Sloterdijk, P. (2010). En el mundo interior del capital. Para una teoría filosófica de la globalización. Madrid, España: Ediciones Siruela S.A.

(2012). Has de cambiar tu vida. Valencia, España: Pre-textos.

(2014) Fiscalidad voluntaria y responsabilidad ciudadana. Aportaciones a un debate filosófico sobre una nueva fundamentación democrática de los impuestos. [Versión de 24Symbols]. Madrid, España: Ediciones Siruela S.A.

Stiglitz, J.E. (2002). El malestar en la globalización. Madrid, España: Taurus.

Wallerstein, I. (2006). Análisis de sistemas-mundo. Una introducción. México D.F., México: Siglo XXI Editores S.A.

Wong, E.M., Peña, J.M. \& Falla, S.O. (2016). La actitud crítica: un aspecto fundamental en la educación. Sophia, 12 (1), 107-114.

Zaid, G. (2014). Los demasiados libros. El Malpensante, 158, 10-15. 